\title{
Influência da temperatura e luminosidade na germinação de sementes das espécies: Selenicereus setaceus, Hylocereus undatus e Hylocereus polyrhizus
}

\author{
Influence of temperature and luminosity in seed germination of species: Selenicereus setaceus, \\ Hylocereus undatus and Hylocereus polyrhizus
Rodrigo Ruths, Lisandro Tomas da Silva Bonome*, Yasmin Tomazi, Diogo José Siqueira, Gabriela Silva Moura e Cláudia Simone Madruga Lima

Universidade Federal da Fronteira Sul, Laranjeiras do Sul, Paraná, Brasil. *Autor para correspondência: lisandrobonome@gmail.com.

Submissão:13/10/2017 | Aceite: 25/02/2019

\begin{abstract}
RESUMO
A pitaia é uma frutífera tropical, pertencente à família Cactaceae, com elevado potencial produtivo, nutritivo, econômico e social para a agricultura familiar, sendo uma cultura ainda pouco conhecida e com escassas informações sobre as condições que afetam a germinação das diferentes espécies de pitaia. Assim, o trabalho teve como objetivo avaliar a influência da temperatura e da luminosidade na germinação de sementes de três genótipos de pitaia. Para avaliar o efeito da temperatura na qualidade fisiológica das sementes foram utilizadas as espécies Selenicereus setaceus (pitaia-do-cerrado), Hylocereus undatus (casca vermelha e polpa branca) e Hylocereus polyrhizus (casca vermelha rosada e polpa vermelha) submetidas à germinação em sete temperaturas $\left(15^{\circ} \mathrm{C}, 20^{\circ} \mathrm{C}, 25^{\circ} \mathrm{C}, 30^{\circ} \mathrm{C}\right.$ e $35^{\circ} \mathrm{C}$, e em temperaturas alternadas: $15-25 \stackrel{\circ}{\circ}$ e 20-30 $\stackrel{\circ}{ }$, todas com fotoperíodo de 12 horas). As avaliações realizadas foram: porcentagem de germinação, índice de velocidade de germinação e tempo para ocorrência de $50 \%$ de germinação. Para a avaliação da influência da luminosidade, as sementes foram acondicionadas em frasco envolto por papel alumínio, a fim de proteger as sementes de qualquer contato com luminosidade, e armazenadas em refrigerador em temperatura de $10 \pm 2{ }^{\circ} \mathrm{C}$ por 365 dias. Após, foram submetidas ao tratamento com e sem luz e mantidas em BOD por 11 dias, quando se realizou a contagem das plântulas normais. As temperaturas para o máximo desempenho fisiológico das sementes das três espécies de pitaia foram de $25^{\circ} \mathrm{C}$ e $20-30^{\circ} \mathrm{C}$. Por outro lado, a temperatura mais desfavorável foi de $35^{\circ} \mathrm{C}$. A espécie Selenicereus setaceus apresentou maior percentual de germinação e vigor em todas as temperaturas avaliadas. As espécies avaliadas demonstraram ser fotoblásticas positivas.
\end{abstract}

PALAVRAS-CHAVE: pitaia, cactaceae, fruta dragão, fruticultura, qualidade fisiológica.

\begin{abstract}
Pitaya is a tropical fruit belonging to the Cactaceae family. With high productive, nutritive, economic and social potential for family farming, it is a still little known culture with scarce information on the conditions affecting the germination of its different species. Thus, this study aimed to evaluate the influence of temperature and luminosity on seed germination of three genotypes of pitaya. To evaluate the effect of temperature on the physiological quality of the seeds, the Selenicereus setaceus, Hylocereus undatus (red peel and white pulp) and Hylocereus polyrhizus were used and (pink red peel and red pulp) submitted to seven germination temperatures $\left(15^{\circ} \mathrm{C}, 20^{\circ} \mathrm{C}, 25^{\circ} \mathrm{C}, 30^{\circ} \mathrm{C}\right.$, and $35^{\circ} \mathrm{C}$, and in alternating temperatures: $15-25^{\circ} \mathrm{C}$ and $20-30^{\circ} \mathrm{C}$, all in a photoperiod of 12 hours). The evaluations were: percentage of germination, germination speed index, and time for occurrence of $50 \%$ of germination. For the evaluation of the influence of the luminosity, the seeds were conditioned in bottle wrapped in aluminum foil to protect the seeds from contact with the luminosity and stored in refrigerator at $10 \pm 2{ }^{\circ} \mathrm{C}$ for 365 days. They were then submitted to the treatment with and without light and kept in BOD for eleven days, when the normal seedling were counted. The temperatures for the maximum physiological performance of the seeds of three species of pitaya were $25^{\circ} \mathrm{C}$ and the alternating $20-30^{\circ} \mathrm{C}$. On the other hand, the unfavorable temperature was $35^{\circ} \mathrm{C}$. The Selenicereus setaceus showed a higher percentage of germination and vigor in all temperatures evaluated. The species evaluated demonstrated to be positive photoblastic.
\end{abstract}

KEYWORDS: pitaya, cactaceae, dragon fruit, fruticulture, physiological quality. 


\section{INTRODUÇÃO}

A pitaia é uma Cactaceae originada de florestas tropicais e subtropicais do México, América Central e América do Sul (ORTIZ \& LIVEIRA 1995, MIZRAHI et al. 1997). A origem etimológica do termo pitaia, significa pitahaya, que faz menção a uma civilização pré-colombiana que habitava algumas ilhas da América Central, os Tainos, e significa fruta escamosa (MERRIAM-WEBSTER 2017).

Essa fruta pode ser consumida in natura, bem como industrializada na forma de sorvete, sucos e saladas, mousse, geleias ou como corantes de doces. Além disso, a fruta pode ser utilizada na indústria farmacêutica como tônico cardíaco, devido à presença da substância captina disponível no fruto, que auxilia na regulação da pressão arterial (DONADIO 2009), combate as doenças relacionadas ao sistema respiratório como bronquite, possui propriedades curativas e protege contra úlceras, gastrites (MOLINA et al. 2009) e tem função antidiabética e anti-inflamatória (HERBACH et al. 2007).

Conforme LE BELLEC et al. (2006), as pitaias são agrupadas em quatro gêneros principais, sendo eles: Stenocereus, Cereus, Selenicereus e Hylocereus. As espécies Hylocereus undatus e Selenicereus megalanthus têm sido as mais cultivadas e comercializadas no mundo (MIZRAHI et al. 1997, NERD et al. 2002). No Brasil, o gênero Hylocereus sp. é o mais cultivado, com produção entre 10 e $30 \mathrm{t} \mathrm{ha}^{-1}$, que pode variar conforme as técnicas de cultivo, a genética e a idade da planta (LAREDO 2016). A região Sudeste é responsável pela maior parte da produção de pitaia (NUNES et al. 2014), com 95,9\% da produção, seguida da região Sul e região Nordeste que representam aproximadamente produção de 2,38 e 1,05\%, respectivamente (CONAB 2016).

No Brasil, essa frutífera é considerada exótica, mesmo sendo encontradas espécies de pitaia nativas em matas de transição e no cerrado brasileiro, principalmente espécies do gênero Selenicereus e Hylocereus, dentre elas a $S$. setaceus, popularmente conhecida como "pitaia-do-cerrado" ou "saborosa" (JUNQUEIRA et al. 2002).

As pitaias podem se reproduzir de forma sexuada ou assexuada. O método mais comumente utilizado pelos produtores para a propagação é a forma assexual, através da estaquia, devido ao manejo simples e rápido para a formação de mudas (ZEE et al. 2004, LE BELLEC et al. 2006). O método de reprodução sexual via propagação seminífera é menos utilizada em cultivos, pois o início da produção é mais tardio, a planta passa pelo período de juvenilidade até completar a maturidade necessária para o início do período reprodutivo, que demora de quatro a seis anos (GUNASENA et al. 2007). Neste contexto, a propagação via seminífera para cultivos comerciais pode tornar-se economicamente inviável (SILVA 2005, SILVA 2014).

No entanto, o método de reprodução sexual proporciona a variabilidade genética necessária para a seleção de características desejáveis em um programa de melhoramento genético, além de possibilitar a investigação científica de fatores que afetam a biologia da germinação (SUÁREZ-ROMÁN et al. 2011). Outra característica importante desse método é a preservação da diversidade dos recursos fitogenéticos e a conservação de espécies por meio de bancos de germoplasma (MARTIN 2002).

Para otimização do método de propagação seminífera é de fundamental importância o conhecimento dos principais fatores que afetam a germinação das sementes, dentre eles, destacam-se a temperatura e a luminosidade. No geral, as sementes apresentam comportamento variável para temperatura (LABOURIAU 1983), existem sementes que apresentam melhor comportamento germinativo quando submetidas à alternância de temperatura, e outras que são favorecidas quando submetidas a temperaturas constantes (SALOMÃO et al. 1995). Embora a luz seja necessária para a germinação de sementes de algumas espécies, a maioria das plantas cultivadas podem germinar na presença ou na ausência de luz, assim como existem espécies em que a germinação é prejudicada pela luz (LABOURIAU 1983, VÁZQUEZ-YANES \& OROZCO-SEGOVIA 1990). Contudo, para a maioria das espécies, a condição de baixa ou ausência de luminosidade provoca alterações no crescimento e no desenvolvimento das plântulas em virtude do estiolamento (BASTOS et al. 2009).

Tanto a temperatura quanto a luminosidade podem ser manipuladas para melhorarem a porcentagem, velocidade e sincronização da germinação com objetivo de aumentar o percentual de plântulas vigorosas. Desse modo, torna-se importante a avaliação de temperaturas alternadas e constantes e da necessidade de luminosidade no teste de germinação para a constatação das melhores condições para a germinação das sementes (LONE et al. 2014a, b).

Ainda que hajam algumas pesquisas sobre a influência da temperatura e da luminosidade na germinação de espécies de pitaia, a maioria dos estudos se concentram na espécie Hylocereus undatus (ALVES et al. 2011, LONE et al. 2014a, LONE et al. 2014b, OLIVEIRA JÚNIOR et al. 2015), havendo ausência ou escassas informações para as espécies Hylocereus polyrhizus e Selenicereus setaceus, esta última nativa do cerrado brasileiro (JUNQUEIRA et al. 2002). 
Assim, o objetivo do trabalho foi avaliar a germinação de sementes de três espécies de pitaia (Selenicereus setaceus, Hylocereus undatus e Hylocereus polyrhizus) sob diferentes condições de temperatura e luminosidade.

\section{MATERIAL E MÉTODOS}

O experimento foi realizado nos Laboratórios de Fisiologia Vegetal e Germinação e Crescimento de Plantas da Universidade Federal da Fronteira Sul (UFFS), campus Laranjeiras do Sul, Laranjeiras do Sul, PR.

As espécies de pitaia utilizadas foram: Selenicereus setaceus (Rizz.), Hylocereus undatus (Haworth) Britton \& Rose (PB) e Hylocereus polyrhizus (F.A.C. Weber) Britton \& Rose (PV), obtidas no município de Jacinto Machado no Vale de Araranguá, no estado de Santa Catarina. Para a extração das sementes dos frutos de pitaia, utilizou-se peneira com água corrente para eliminar os resíduos da polpa e reter somente as sementes, as quais posteriormente, foram colocadas para secar à sombra sobre papel toalha durante 48 horas. Em seguida, foi determinado o teor de água das sementes pelo método da estufa a $105{ }^{\circ} \mathrm{C}$ por 24 horas de acordo com BRASIL (2009).

Para o teste de germinação foram utilizadas quatro repetições de 50 sementes, totalizando 200 sementes para cada espécie. As sementes foram colocadas em caixas tipo gerbox sobre papel mata-borrão umedecido com água 2,5 vezes a massa do papel (BRASIL 2009). As caixas foram acondicionadas em BOD e submetidas aos seguintes tratamentos: temperaturas constantes de $15{ }^{\circ} \mathrm{C}, 20^{\circ} \mathrm{C}, 25^{\circ} \mathrm{C}, 30^{\circ} \mathrm{C}$ e $35^{\circ} \mathrm{C}$, e temperaturas alternadas de $15-25^{\circ} \mathrm{C}$ e $20-30^{\circ} \mathrm{C}$, com fotoperíodo de 12 horas. No $11^{\circ}$ dia após o início do teste foram contabilizadas as plântulas consideradas normais (BRASIL 2009). As avaliações para o índice de velocidade de germinação (IVG) ocorreram por meio de contagens diárias de plântulas normais a partir do surgimento da primeira plântula normal até que o número de plântulas fosse constante, sendo o IVG calculado pelo somatório do número de plântulas normais a cada dia, dividido pelo número de dias decorridos à formação da plântula (MAGUIRE 1962).

Por meio dos dados obtidos no índice de velocidade de germinação foi determinado o tempo necessário para ocorrência de 50\% de germinação (T50), conforme proposto por GUIMARÃES (2000).

$O$ delineamento experimental foi inteiramente casualizado, em esquema fatorial $3 \times 7$, sendo as três espécies: S. setaceus, $H$. undatus e $H$. polyrhizus, e as temperaturas: $15^{\circ} \mathrm{C}, 20^{\circ} \mathrm{C}, 25^{\circ} \mathrm{C}, 30^{\circ} \mathrm{C}$ e $35^{\circ} \mathrm{C}$, e as alternadas $15-25 \stackrel{\circ}{\circ}$ e $20-30 \stackrel{\circ}{\circ}$. Foram utilizadas quatro repetições por tratamento, sendo os dados submetidos à análise de variância pelo software SISVAR, e as médias comparadas pelo teste de Tukey a $5 \%$ de probabilidade (FERREIRA 2011).

Outro experimento foi conduzido a fim de verificar a resposta das sementes à luminosidade e determinar se são fotoblásticas positivas ou negativas. Para tanto, as sementes foram acondicionadas em frasco envolto em papel alumínio e armazenadas em refrigerador em temperatura $10 \pm 2{ }^{\circ} \mathrm{C}$ por aproximadamente um ano, visando garantir que o fitocromo estivesse na forma inativa. $O$ experimento foi constituído de quatro repetições por tratamento, com 50 sementes em cada unidade experimental composta por uma caixa do tipo gerbox com duas camadas de papel mata-borrão umedecidos com 2,5 vezes sua massa em água destilada. As sementes foram dispostas nas caixas gerbox em uma sala com iluminação verde para evitar a ativação do fitocromo das sementes. As caixas foram colocadas em BOD a temperatura de $25^{\circ} \mathrm{C}$ e fotoperíodo de 12 horas, condição escolhida conforme dados obtidos no experimento anterior. No tratamento sem luz, as unidades experimentais foram envoltas em duas camadas de papel alumínio. A contagem das plântulas normais foi realizada no $11^{\circ}$ dia após a implantação do experimento.

$O$ delineamento estatístico utilizado foi o inteiramente casualizado, em esquema fatorial $3 \times 2$, sendo três espécies: $S$. setaceus, $H$. undatus e $H$. polyrhizus e duas condições de luminosidade: ausente e presente. Os dados foram submetidos à análise de variância pelo software SISVAR, e as médias comparadas pelo teste de Tukey a 5\% de significância (FERREIRA 2011).

\section{RESULTADOS E DISCUSSÃO}

Os teores de água encontrados nas sementes das três espécies de pitaia estudadas foram: 8,53\%, $8,12 \%$ e $7,21 \%$ para $H$. polyrhizus, $H$. undatus e $S$. setaceus, respectivamente. Estes resultados indicam que as sementes das espécies de pitaia estudadas são ortodoxas, ou seja, podem ser desidratadas a níveis baixos de umidade ( 5 a $7 \%$ de umidade) e armazenadas em ambientes de baixas temperaturas. ROMAN et al. (2012) encontraram percentuais de umidade entre 6 a 11\%, com valor médio de 8,9\% para semente de Selenicereus megalanthus (Haw.) Britt \& Rose. HERNÁNDEZ (2013) classificou 17 genótipos de Hylocereus spp. e 3 genótipos de Stenocereus spp. como sementes ortodoxas, e encontrou grau de umidade médio 
para sementes destas espécies de 9,43\%.

Para a germinação das sementes de pitaia foi observada interação entre os fatores espécie e temperatura (Tabela 1).

Tabela 1. Porcentagem de germinação (\%) de sementes das espécies: Selenicereus setaceus, Hylocereus polyrhizus e Hylocereus undatus em diferentes temperaturas.

Table 1. Percentage of seed germination (\%) of the species: Selenicereus setaceus, Hylocereus polyrhizus and Hylocereus undatus at different temperatures.

\begin{tabular}{llll}
\hline Temp. $\left({ }^{\circ} \mathrm{C}\right)$ & S. setaceus $(\%)$ & H. polyrhizus $(\%)$ & H. undatus $(\%)$ \\
\hline 15 & $96 \mathrm{Aa}$ & $67 \mathrm{Cc}$ & $83 \mathrm{Ba}$ \\
20 & $100 \mathrm{Aa}$ & $88 \mathrm{Bab}$ & $92 \mathrm{ABa}$ \\
25 & $100 \mathrm{Aa}$ & $97 \mathrm{Aa}$ & $94 \mathrm{Aa}$ \\
30 & $100 \mathrm{Aa}$ & $97 \mathrm{Aa}$ & $91 \mathrm{Aa}$ \\
35 & $92 \mathrm{Aa}$ & $67 \mathrm{Bc}$ & $61 \mathrm{Bb}$ \\
$15-25$ & $99 \mathrm{Aa}$ & $81 \mathrm{Bb}$ & $88 \mathrm{Ba}$ \\
$20-30$ & $100 \mathrm{Aa}$ & $94 \mathrm{ABab}$ & $89 \mathrm{Ba}$ \\
\hline
\end{tabular}

Médias seguidas da mesma letra, na coluna (minúscula) ou linha (maiúscula), não diferem estatisticamente entre si pelo teste de Tukey a $5 \%$ de probabilidade.

De maneira geral, as sementes de $S$. setaceus apresentaram maior percentual de germinação entre as espécies estudadas, independentemente da temperatura utilizada. Contudo, nas temperaturas constantes de 25 e $30^{\circ} \mathrm{C}$ e na alternada de $20-30^{\circ} \mathrm{C}$ não foram observadas diferenças significativas para a porcentagem de germinação entre as espécies, sendo essa a faixa de temperatura mais propícia para a germinação das três espécies. SIMÃO et al. (2007) também relataram maior porcentagem de germinação de sementes de $H$. setaceus quando submetidas a temperaturas entre 25 e $30{ }^{\circ} \mathrm{C}$. Esses resultados corroboram os encontrados por ALVES et al. (2011) que avaliaram diferentes substratos e temperaturas na germinação de sementes de $H$. undatus, evidenciando maior percentual de germinação quando foi utilizado o substrato rolo de papel, à temperatura de $25^{\circ} \mathrm{C}$. Pesquisa realizada por OLIVEIRA JÚNIOR et al. (2015) avaliando diferentes volumes de água no substrato e temperaturas na germinação e no vigor de sementes de $H$. undatus também evidenciaram que a temperatura de $25{ }^{\circ} \mathrm{C}$ e a umidade de substrato 2,5 e 3,5 vezes o peso do papel seco foram as mais adequadas para o máximo desempenho da qualidade fisiológica das sementes.

Nas temperaturas extremas, $15{ }^{\circ} \mathrm{C}$ e $35^{\circ} \mathrm{C}$, as espécies $H$. polyrhizus e $H$. undatus apresentaram germinação inferior à observada para a espécie $S$. setaceus. A baixa temperatura afeta a velocidade e a porcentagem de germinação, influenciando negativamente na absorção de água pelas sementes e, consequentemente, reduzindo as reações bioquímicas e fisiológicas que determinam a germinação (CARVALHO \& NAKAGAWA 2012). Contudo, altas temperaturas podem aumentar a fluidez de lipídeos e reduzir a estabilidade das membranas celulares, ocasionando perdas de íons e até mesmo a ruptura de membranas (TAIZ \& ZEIGER 2013), consequentemente prejudicando a qualidade fisiológica das sementes (MARCOS-FILHO 2015).

A espécie $H$. polyrhizus foi a que apresentou maior sensibilidade a variação da temperatura em relação à ótima, que ficou entre $25^{\circ} \mathrm{C}$ e $30^{\circ} \mathrm{C}$. Temperaturas acima ou abaixo desses valores foram responsáveis pela redução na germinação das sementes, embora as temperaturas constante de $20{ }^{\circ} \mathrm{C} e$ alternada de 20-30 ํ $\mathrm{C}$ não tenham diferido daquelas consideradas ótimas. LONE et al. (2014a), observaram resultados semelhantes aos obtidos no presente trabalho para a espécie $H$. polyrhizus, no entanto, as temperaturas constante de $15{ }^{\circ} \mathrm{C}$ e a alternada $25-35^{\circ} \mathrm{C}$ não reduziram a porcentagem de germinação para esta espécie. Já a espécie $H$. undatus se mostrou mais tolerante a variação de temperatura do que a espécie $H$. polyrhizus, apresentando redução na germinação somente quando exposta a temperatura alta de $35{ }^{\circ} \mathrm{C}$. A espécie $S$. setaceus apresentou germinação superior a $90 \%$ em todas as temperaturas testadas, demonstrando maior tolerância as variações de temperatura para a germinação.

A ampla faixa de temperatura na qual as sementes de $S$. setaceus apresentam germinação superior a $90 \%$, de 15 a $35{ }^{\circ} \mathrm{C}$, pode ser um mecanismo evolutivo adaptativo que garante maiores chances de sobrevivência em comparação com outras espécies que apresentam estreitos limites de temperatura para a germinação. As espécies com potencial de resistência à amplas variações de temperatura são denominadas de euritérmicas (LEMES \& LOPES 2012).

Observou-se que, enquanto a germinação das sementes de pitaia da espécie $S$. setaceus não foi 
influenciada pela variação na temperatura de germinação, o vigor medido pelo índice de velocidade de germinação foi severamente prejudicado (Tabela 2), reduzindo os valores mais de $100 \%$ em temperaturas abaixo de $25^{\circ} \mathrm{C}$ e acima de $30^{\circ} \mathrm{C}$. Segundo CUSTÓDIO (2005), os testes de vigor são mais sensíveis para detectar perdas na qualidade de sementes do que o teste de germinação, sendo utilizado para evidenciar diferenças significativas na qualidade fisiológica de lotes de sementes com germinação semelhante.

Tabela 2. Índices de velocidade de germinação para as três espécies: S. setaceus, $H$. polyrhizus e $H$. undatus em diferentes temperaturas.

Table 2. Germination speed indexes for the three species: S. setaceus, $H$. polyrhizus and $H$. undatus at different temperatures.

\begin{tabular}{lccc}
\hline Temp. $\left({ }^{\circ} \mathrm{C}\right)$ & S. setaceus & H. polyrhizus & H. undatus \\
\hline 15 & $6,36 \mathrm{Ab}$ & $4,42 \mathrm{Ad}$ & $6,35 \mathrm{Ad}$ \\
20 & $10,21 \mathrm{Ab}$ & $8,89 \mathrm{Ac}$ & $10,13 \mathrm{Acd}$ \\
25 & $23,72 \mathrm{Aa}$ & $21,77 \mathrm{Aab}$ & $19,12 \mathrm{Ba}$ \\
30 & $19,93 \mathrm{Aa}$ & $15,98 \mathrm{Bb}$ & $14,10 \mathrm{Bbc}$ \\
35 & $10,21 \mathrm{Ab}$ & $7,41 \mathrm{ABcd}$ & $6,15 \mathrm{Bd}$ \\
$15-25$ & $8,01 \mathrm{Ab}$ & $8,89 \mathrm{Ac}$ & $9,31 \mathrm{Ad}$ \\
$20-30$ & $19,65 \mathrm{Aa}$ & $18,89 \mathrm{Aab}$ & $18,40 \mathrm{Aab}$ \\
\hline
\end{tabular}

Médias seguidas da mesma letra, na coluna (minúscula) ou linha (maiúscula), não diferem estatisticamente entre si pelo teste de Tukey a $5 \%$ de probabilidade.

Da mesma maneira que o percentual de germinação, as temperaturas constante de $25^{\circ} \mathrm{C}$ e alternada de $20-30{ }^{\circ} \mathrm{C}$ foram as que proporcionaram o maior IVG nas sementes de pitaia, para todas as espécies avaliadas. Estes resultados sugerem que as temperaturas ótimas para a germinação e o vigor das sementes de pitaia das espécies, $H$. polyrhizus, S. setaceus e $H$. undatus são de $25{ }^{\circ} \mathrm{C}$ e $20-30{ }^{\circ} \mathrm{C}$. Resultados semelhantes foram encontrados por NOBEL (1988), ROJAS-ARÉCHIGA \& VÁZQUEZ-YANES (2000), LONE et al. (2007) e MEIADO et al. (2010) para espécies de cactáceas, em que a temperatura ideal para germinação e índice de velocidade de germinação foi em torno de $25 \stackrel{\circ}{\circ}$.

O efeito da temperatura alternada na germinação e no vigor de sementes de cactáceas ainda não está totalmente elucidado, visto que a maioria dos estudos utilizam temperaturas constantes. As escassas pesquisas que utilizam temperaturas alternadas não evidenciaram um significante acréscimo na germinação e no vigor das sementes quando comparado às temperaturas constantes (ROJAS-ARÉCHIGA \& VÁZQUEZYANES 2000).

Em geral, a espécie $S$. setaceus foi mais tolerante às variações de temperatura do que as demais espécies. $H$. polyrhizus e $H$. undatus apresentaram comportamento semelhante, exceção na temperatura de $25^{\circ} \mathrm{C}$ em que a espécie $H$. polyrhizus apresentou velocidade de germinação superior à da $H$. undatus. Os dados de percentual de germinação em função do tempo apresentaram distribuição normal e foram utilizados para a constituição de uma curva de regressão modelo de Gauss. Conforme esta análise, observou-se que as sementes de $S$. setaceus realizaram o processo de germinação entre o primeiro e o terceiro dia após a semeadura (Figura 1). A germinação das sementes de $H$. polyrhizus e $H$. undatus se completou até o quarto e quinto dia, respectivamente. Estes resultados evidenciam que a velocidade e a uniformidade de germinação são maiores na espécie $S$. setaceus quando comparado as espécies $H$. polyrhizus e $\mathrm{H}$. undatus.

O menor tempo para ocorrência de $50 \%$ de germinação (T50) foi obtido para as temperaturas constantes de 25 e $30^{\circ} \mathrm{C}$ e alternada de $20-30{ }^{\circ} \mathrm{C}$ (Tabela 3). Os menores valores de T50 implica em menor tempo de exposição das sementes às condições adversas do ambiente no período pré-germinativo, podendo dar origem a plântulas mais vigorosas, com maiores chances na competição por recursos como água, luz e nutrientes minerais (FENNER 2000). LONE et al. (2014a) encontraram resultados similares para TMG (tempo médio de germinação) para os genótipos $H$. undatus e $H$. polyrhizus. Em $H$. undatus, o menor TMG (7,1 dias) foi obtido na temperatura de $25^{\circ} \mathrm{C}$, não diferindo das temperaturas de 30 e $20-30^{\circ} \mathrm{C}$. Em $\mathrm{H}$. polyrhizus o menor TMG foi observado a $25^{\circ} \mathrm{C}\left(4,4\right.$ dias), não diferindo da temperatura de $20^{\circ} \mathrm{C}$ e $30^{\circ} \mathrm{C}$ (4,5 e 4,9 dias, respectivamente). A partir dos parâmetros avaliados, a espécie $S$. setaceus mostrou-se adaptada a uma ampla faixa de temperatura, sugerindo uma maior capacidade de distribuição nas diferentes regiões de cerrado, bem como em outras áreas brasileiras em comparação as outras espécies avaliadas. 

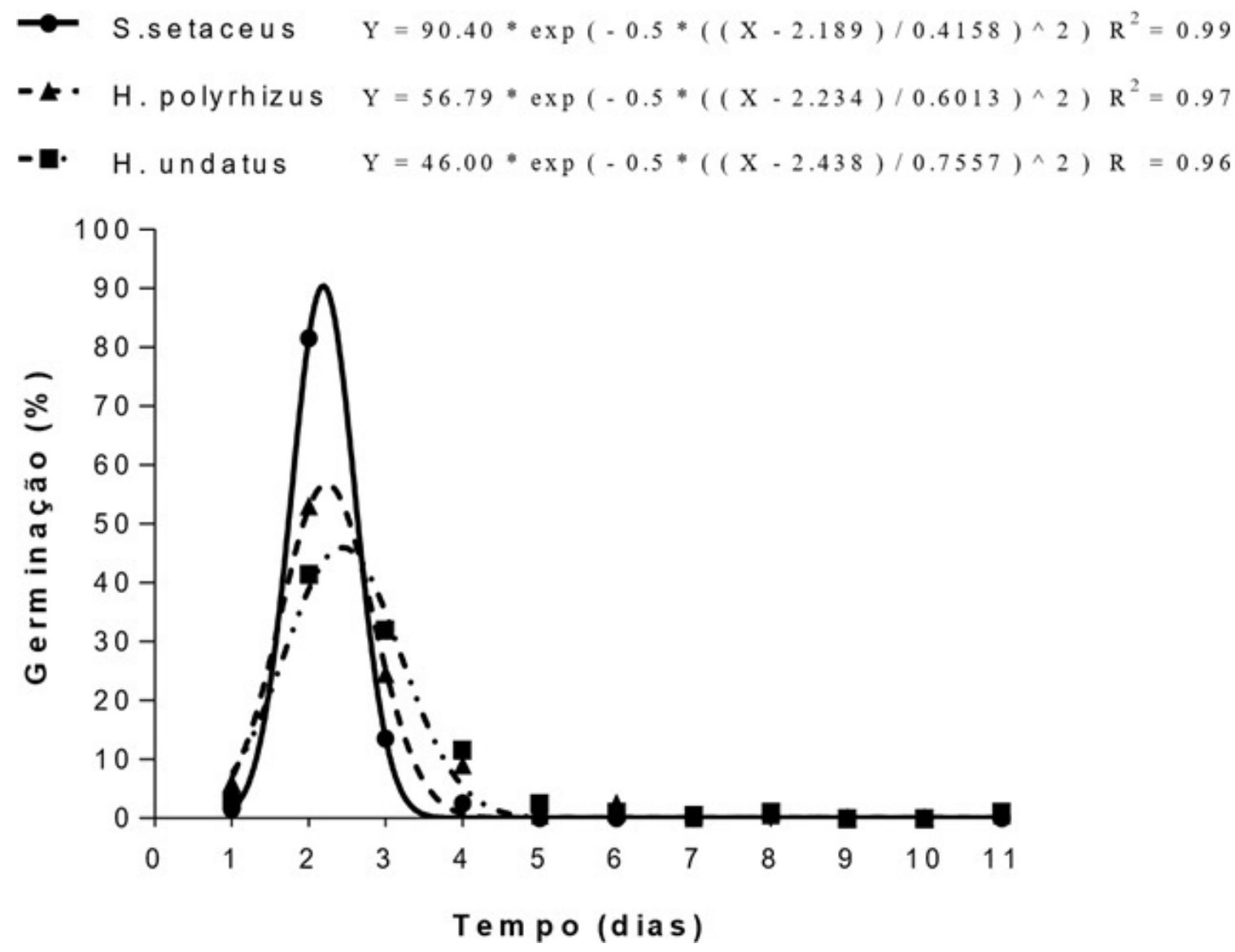

Figura 1. Distribuição da porcentagem de germinação de sementes das espécies $S$. setaceus, $H$. polyrhizus e $H$. undatus submetidas a temperatura de $25^{\circ} \mathrm{C}$ até $011^{\circ}$ dia após a semeadura. Os símbolos representam a média geral dos tratamentos. A curva de regressão representa o modelo de Gauss em que $\mathrm{Y}$ representa sementes germinadas (\%) a um determinado período de tempo (dias) $\mathrm{X}$.

Figure 1Distribution of seed germination percentage of S.setaceus, $\mathrm{H}$. polyrhizus, and $\mathrm{H}$. undatus species submitted to temperature from $25{ }^{\circ} \mathrm{C}$ to $11^{\circ}$ day after sowing. Symbols represent the general average of treatments. The curve represents a model of Gauss, in which $Y$ represents germinated seeds (\%) at a given period of time (days) $X$.

Tabela 3. Tempo para ocorrência de $50 \%$ de germinação de semente das espécies: S.setaceus, $H$. polyrhizus e $\mathrm{H}$. undatus em diferentes temperaturas.

Table 3. Time for $50 \%$ seed germination of the species: S.setaceus, $H$. polyrhizus and $H$. undatus at different temperatures.

\begin{tabular}{llll}
\hline Temp. $\left({ }^{\circ} \mathrm{C}\right)$ & S. setaceus & H. polyrhizus & H. undatus \\
\hline 15 & $8,53 \mathrm{Ac}$ & $8,48 \mathrm{Ad}$ & $6,84 \mathrm{Bc}$ \\
20 & $5,23 \mathrm{Ab}$ & $5,21 \mathrm{Ac}$ & $5,36 \mathrm{Ab}$ \\
25 & $2,42 \mathrm{Aa}$ & $2,44 \mathrm{Aa}$ & $3,04 \mathrm{Aa}$ \\
30 & $3,05 \mathrm{Aa}$ & $3,65 \mathrm{Aab}$ & $3,31 \mathrm{Aa}$ \\
35 & $5,12 \mathrm{Ab}$ & $5,12 \mathrm{Ac}$ & $4,89 \mathrm{Ab}$ \\
$15-25$ & $7,15 \mathrm{Ac}$ & $5,02 \mathrm{Bbc}$ & $5,02 \mathrm{Bb}$ \\
$20-30$ & $2,57 \mathrm{Aa}$ & $2,56 \mathrm{Aa}$ & $2,60 \mathrm{Aa}$
\end{tabular}

Médias seguidas da mesma letra, na coluna (minúscula) ou linha (maiúscula), não diferem estatisticamente entre si pelo teste de Tukey a $5 \%$ de probabilidade.

Para a determinação da fotoblastia, observou-se que as sementes do tratamento sem radiação apresentaram percentual de germinação significativamente inferior àquelas do tratamento com radiação (Tabela 4).

As três espécies de pitaia avaliadas demonstraram ser fotoblásticas positivas, sendo as sementes de $S$. setaceus mais dependentes de luz para a germinação do que as de $H$. polyrhizus que, por sua vez, foram 
mais dependentes que as de $H$. undatus.

Tabela 4. Percentual de germinação de sementes de S. setaceus, H. polyrhizus e H. undatus em diferentes condições de exposição à radiação.

Table 4. Percentage of seed germination of the species: S. setaceus, H. polyrhizus and H. undatus under different conditions of radiation exposure.

\begin{tabular}{llll}
\hline Fotoperíodo & S. setaceus $(\%)$ & H. polyrhizus (\%) & H. undatus (\%) \\
\hline Com luz & $72 \mathrm{Aa}$ & $69 \mathrm{Aa}$ & $75 \mathrm{Aa}$ \\
Sem luz & $1 \mathrm{Bb}$ & $7 \mathrm{Bb}$ & $46 \mathrm{Ab}$ \\
\hline
\end{tabular}

Médias seguidas da mesma letra, na coluna (minúscula) ou linha (maiúscula), não diferem estatisticamente entre si pelo teste de Tukey a $5 \%$ de probabilidade.

As diferentes espécies de pitaia integram a maior parte das plantas com sementes pequenas e com poucas reservas que, em sua grande maioria, são fotoblásticas positivas (MAJEROWICZ \& PERES 2008). As sementes pequenas não possuem reservas suficientes para sustentar prolongados períodos de crescimento de plântulas no escuro, necessitando de luz para germinação (JANKOWSKA-BLASZCZUK \& DAWS 2007). O controle da germinação pela luz é regulado por um pigmento proteico denominado de fitocromo. Este pigmento apresenta duas formas interconversíveis: uma que absorve a luz vermelha e azul e outra que absorve a luz vermelho-distante (BORTHWICK et al. 1952). Quando absorvida luz vermelha e/ou luz azul ocorre a fotoconversão da forma do fitocromo Fv (inativo) para Fve (ativo), induzindo respostas metabólicas e de desenvolvimento do vegetal. Por outro lado, a reversão de Fve para Fv é estimulada por comprimento de onda no vermelho distante e também pelo escuro (NAGY \& SCHAFER 2002).

A detecção da luz pelas sementes tem um importante papel na sobrevivência das plantas, prevenindo a ocorrência de germinação em condições inadequadas para o estabelecimento das mesmas. A habilidade nesse reconhecimento permite que as sementes controlem onde e quando a germinação poderá ocorrer, aumentando as probabilidades de sucesso no estabelecimento das plantas (FENNER \& THOMPSON 2005).

\section{CONCLUSÃO}

Nas condições do presente trabalho, as temperaturas para o melhor desempenho fisiológico das sementes das espécies de pitaia Selenicereus setaceus, Hylocereus undatus e Hylocereus polyrhizus foram as temperaturas constante de $25^{\circ} \mathrm{C}$ e alternada de 20-30 ${ }^{\circ} \mathrm{C}$. Por outro lado, a temperatura mais desfavorável foi de $35^{\circ} \mathrm{C}$. A espécie $S$. setaceus apresentou maior percentual de germinação e vigor em todas as temperaturas avaliadas. As espécies avaliadas demonstraram ser fotoblásticas positivas.

\section{REFERÊNCIAS}

ALVES CZ et al. 2011. Adequação da metodologia para o teste de germinação de sementes de pitaia vermelha. Ciência Rural 41: 779-784.

BASTOS DC et al. 2009. Estiolamento, incisão na base da estaca e uso de ácido indolbutírico na propagação da caramboleira por estacas lenhosas. Ciência e Agrotecnologia 33: 313-318.

BORTHWICK HA et al. 1952. A reversible photoreaction controlling seed germination. Proceedings of the National Academy of Sciences 38: 662-666.

BRASIL. 2009. Ministério da Agricultura, Pecuária e Abastecimento. Regras para análise de sementes. Ministério da Agricultura, Pecuária e Abastecimento. Secretaria de Defesa Agropecuária. Brasília: MAPA/ACS. 399 p.

CARVALHO NM \& NAKAGAWA J. 2012. Sementes: ciência, tecnologia e produção. 5.ed. Jaboticabal: FUNEP. 590p.

CUSTÓDIO CC. 2005. Testes rápidos para avaliação do vigor de sementes: uma revisão. Colloquium Agrariae 1: $29-41$.

DONADIO LC. 2009. Pitaya. Revista Brasileira de Fruticultura 31: 637-979.

FENNER M. 2000. Seeds: the ecology of regeneration in plant communities. Wallingford: CABI Publishing. 320p.

FENNER M \& THOMPSON K. 2005. The ecology of seeds. Cambridge: Cambridge University Press. 250p.

FERREIRA DF. 2011. Sisvar: A computer statistical analysis system. Ciência e Agrotecnologia 35: 1039-1042.

GUIMARÃES RM. 2000. Tolerância à dessecação e condicionamento fisiológico em sementes de cafeeiro (Coffea arabica L.). Tese (Doutorado em Fitotecnia). Lavras: UFLA. 180p.

GUNASENA HPM et al. 2007. Dragon Fruit-Hylocereus undatus (Haw.) Britton and Rose In: Field Manual for Extension Workers. Wijerama Mawatha: Sri Lanka Council for Agricultural Policy. p.111-138.

HERBACH KM et al. 2007. Effects of processing and store on juice color and betacyanin stability of purple pitaya (Hylocereus polyrhizus) juice. European Food Research and Technology 224: 649-658.

HERNÁNDEZ OG. 2013. Germinación y longevidad de semillas de genotipos de Pitahaya (Hylocereus spp) y Pitahaya (Stenocereus spp). Dissertação (Mestrado em Ciências). Colegio de postgraduados - Institucion de enseñansa e 
investigacion en ciências agrícolas, Montecillo. 94p.

JANKOWSKA-BLASZCZUK M \& DAWS MI. 2007. Impact of red : far red ratios on germination of temperate forest herbs in relation to shade tolerance, seed mass and persistence in the soil. Functional Ecology 21: 1055-1062.

JUNQUEIRA KP et al. 2002. Informações preliminares sobre uma pitaya (Selenicereus setaceus Rizz.) nativa do cerrado. In: $17^{\circ}$ Congresso Brasileiro de Fruticultura. Anais... Belém: Sociedade Brasileira de Fruticultura. CD-ROM.

LABOURIAU LG. 1983. A germinação das sementes. Washington: Secretaria da OEA. 173p.

LAREDO RR. 2016. Épocas de coleta e tipos de incisão no cladódio para propagação de pitaia vermelha de polpa branca. Tese (Doutorado em Agronomia). Lavras: UFLA. 83p.

LE BELLEC F et al. 2006. Pitahaya (Hylocereus spp.): a new fruit crop, a market with a future. Fruits 61: 237-250.

LEMES EQ \& LOPES JC. 2012. Temperaturas cardinais para germinação de sementes e desenvolvimento de plântulas de paineira. Scientia Forestalis 40: 179-186.

LONE AB et al. 2007. Germinação de Melocactus bahiensis (cactaceae) em diferentes substratos e temperaturas. Scientia Agraria 8: 365-369.

LONE AB et al. 2014a. Temperatura na germinação de sementes de genótipos de pitaya. Semina: Ciências Agrárias 35: 2251-2258.

LONE AB et al. 2014b. The effects of light wavelength and intensity on the germination of pitaya seed genotypes. Australian Journal of Crop Science 8: 1475-1480.

MAGUIRE JD. 1962. Speed of germination aid in selection and evaluation for seedling emergence and vigor. Crop Science 2: 176-177.

MAJEROWICZ N \& PERES LEP. 2008. Fotomorfogênese em plantas. In: KERBAUY GB. Fisiologia Vegetal. 2.ed. Rio de Janeiro: Guanabara Koogan S.A. 431p.

MARCOS-FILHO J. 2015. Fisiologia de sementes de plantas cultivadas. Londrina: ABRATES. 659p.

MARTIN A. 2002. Los marcadores genéticos en la Mejora Vejetal. In: NUEZ F et al. (Eds.). Genómica y Mejora Vejetal. Sevilla: Mundi-Prensa. p.37-64.

MEIADO MV et al. 2010. Seed germination responses of Cereus jamacaru DC. ssp. jamacaru (Cactaceae) to environmental factors. Plant Species Biology 25: 120-128.

MERRIAM-WEBSTER. 2017. Enciclopédia Britânica. Disponível em: http://www.merriamwebster.com/dictionary/pitaha ya. Acesso em: 05 abr. 2017.

MIZRAHI YA et al. 1997. Cacti as crops. Horticultural Review 18: 291-320.

MOLINA DJ et al. 2009. Producción y expertación de la pitahaya hacia el mercado europeo. Monografia (Especializacion en Finanzas). Guayaquil: Facultad de Economía y Negocios. 115p.

NAGY F \& SCHÄFER E. 2002. Phytochromes control photomorphogenesis by differentially regulated, interacting signaling pathways in higher plants. Review of Plant Biology 53: 329-355.

NERD A et al. 2002. Fruit of vine and columnar cacti. In: NOBEL PS. (Ed.). Cacti: biology and uses. Los Angeles: UCLA. p.254-262.

NOBEL PS. 1988. Environmental biology of agaves and cacti. New York: Cambridge University Press. 270p.

NUNES EN et al. 2014. Pitaya (Hylocereus sp.): uma revisão para o Brasil. Gaia Scientia 8: 90-98.

OLIVEIRA JÚNIOR JL et al. 2015. Umedecimento do substrato e temperatura na germinação e vigor de sementes de pitaya. Comunicata Scientiae 6: 282-290.

ORTIZ HYD \& LIVERA MM. 1995. La pitahaya (Hylocereus spp): Recurso genético de América. In: Congreso Nacional, 6 Y Internacional Sobre El Conocimiento Y Aprovechamiento Del Nopal, 4. Guadalajara. Memórias... p.191-194.

CONAB. 2016. Companhia Nacional de Abastecimento. Programa Brasileiro de Modernização do Mercado Hortigranjeiro. Disponível em: http://dw.prohort.conab.gov.br/pentaho/Prohort. Acesso em: 02 dez. 2016.

ROJAS-ARÉCHIGA M \& VÁZQUEZ-YANES C. 2000. Cactus seed germination: a review. Journal of Arid Environments 44: 85-104.

ROMAN RSS et al. 2012. Caracterización morfoanatómica y fisiológica de semilla sexual de pitahaya amarilla Selenicereus megalanthus (Haw.) Britt \& Rose. Revista de La Asociación Colombiana Ciencias Biológicas 24: 97-111.

SALOMÃO AN et al. 1995. The Effect of Temperature on Seed Germination of Four Dalbergia nigra Fr. Allem. - Leguminosae Trees. Revista Árvore 19: 588-594.

SIMÃO E et al. 2007. The Epiphytic Cactaceae Hylocereus setaceus (Salm-Dick ex DC.) Ralf Bauer Seed Germination is Controlled by Light and Temperature. Brazilian Archives of Biology and Technology 50: 655-662.

SILVA ACC. 2014. Pitaya: Melhoramento e produção de mudas. Tese (Doutorado em Produção Vegetal). Jaboticabal: UNESP. 132p.

SILVA MTH. 2005. Propagação sexuada e assexuada da pitaya vermelha (Hylocereus undatus Haw). TCC (Graduação em Agronomia). Jaboticabal: UNESP. 44p.

SUÁREZ-ROMÁN RS et al. 2011. Evaluación de métodos de propagación en pitahaya amarilla Selenicereus megalanthus (Haw.) Britt and Rose y pitahaya roja Hylocereus polyrhizus (Haw.) Britt and Rose. Tese (Magister en Ciencias Agrarias). Palmira: UNC. 280p.

TAIZ L \& ZEIGER E. 2013. Fisiologia vegetal. 5.ed. Porto Alegre: Artmed. 918p.

VÁZQUEZ-YANES C \& OROZCO-SEGOVIA A. 1990. Ecological significance of light controlled seed germination in two contrasting tropicais habitats. Oecologia 83: 171-175.

ZEE F et al. 2004. Pitaya (Dragon Fruit, Strawberry Pear). Fruits and Nuts 9: 1-3. 